\title{
ОСОБЕННОСТИ РАСПРОСТРАНЕНИЯ НЕРАВНОВЕСНЫХ ФОНОНОВ В УСЛОВИЯХ СИЛЬНОГО РЕЗОНАНСНОГО РАССЕЯНИЯ
}

I. DOLINDO, I. SILDOS. MITTETASAKAALULISTE FOONONITE LEVIKU ISEARASUSED TUGEVA RESONANTSE HAJUMISE TINGIMUSTES

I. DOLINDO, I. SILDOS. PECULIARITIES OF NONEQUILIBRIUM PHONON PROPAGATION UNDER THE CONDITIONS OF STRONG RESONANCE SCATTERING

\author{
(Представил К. К. Ребане)
}

\section{Введение}

Свет, проходящий через среду, как правило, вызывает возбуждение фононной системы: в области прозрачности имеют место релеевские процессы, а при поглощении часть световой энергии, благодаря безызлучательным переходам, преобразуется в тепловое движение частиц среды. Изучение этих процессов стало особенно актуальным в связи с применением в качестве источников света лазеров, в частности импульсных, так как в последних легко достигаются высокие пиковые мощности излучения. При конструировании современных лазерных оптических приборов, во избежание локальных механических и термических перегрузок, необходимо знать особенности их возникновения и развития в среде под действием лазерного света. Кроме того, вызванные фононные неравновесности могут быть использованы как основа работы оптических приборов (акустооптические модуляторы, пространственные решетки, оптические затворы).

В экспериментальных нсследованиях фононные неравновесности обычно создаются пленочными омнческими нагревателями, сверхпроводящими туннельными диодами или оптическим возбуждением среды. Детектироваться фононы могут болометрами, туннельными диодами, а также оптическими методами (люминесценция, поглощение, рассеяние) $\left[{ }^{1}\right]$.

Режим распространения созданной фононной неравновесности зависит от соотношения процессов рассеяния фононов на дефектах и фононфононного взаимодействия. В фонон-фононном взаимодействии при малых числах заполнения неравновесных фононов учитывается только ангармонический распад, а при числах заполнения $\sim 1$ существен и процесс слияния фононов. Равновесие этих двух процессов ведет к установлению локальной температуры в горячей области кристалла.

При доминировании процессов упругого рассеяния на дефектах кристалла режим распространения неравновесных фононов всегда имеет диффузионный характер. Фонон-фононное взаимодействие усложняет диффузию: в зависимости от соотношения времен рассеяния и распада, от начального распределения фононов реализуются различные режимы диффузионного распространения фононов (простая диффузия, квазидиффузия, нелокальная теплопроводность $[2,3])$. 


\section{Результаты эксперимента}

Использованная в данной работе методика подробно описана в $\left[{ }^{4}\right]$. Пакет неравновесных фононов создавался в цилиндрическом объеме с диаметром $\sim 0,2$ мм в ходе безызлучательной релаксации после возбуждения $\mathrm{NO}_{2}^{-}$-примесей лазерным импульсом света в полосе примесного поглощения. Количество поглощенной кристаллом энергии контролировалось как разность энергии светового импульса до и после прохождения им кристалла. Пробными импульсами другого лазера в стробоскопическом режиме измерялись кинетики пропускания кристалла на длине волны чисто электронной линии поглощения $\mathrm{NO}_{2}^{-}$. Измерения проведены для четырех различных концентраций примесей, при этом количество поглощенной кристаллом энергии варьировалось в $\sim 15$ раз.

Обработка измеренных кинетик проведена по модели, предложенной в $\left[{ }^{4}\right]$, в которой полагается, что относительное изменение интенсивности поглощения чисто электронной линии примеси $\Delta x / x \sim \int \Delta n(\omega) \varrho(\omega) d \omega$, где $\Delta n(\omega)$ - изменение чисел заполнения фононов, $\varrho(\omega)$ - плотность фононных состояний. Мы предполагаем, что в возбужденной области для основной части фононного распределения устанавливается равновесие (т. е. температура), и изменение поглощения описывается фактором Дебая-Валлера. Решая нестационарное уравнение диффузии из цилиндрического объема $\left[{ }^{4}\right]$, подбираем коэффициент диффузии $D$, которым наилучшим способом аппроксимируется временной ход $\Delta x(t)$ при данной энергии накачки $W_{\text {н. }}$.

\section{Обсуждение}

Из результатов эксперимента (рис. 1) следует, что $D^{-1}$ прямо пропорционален концентрации $\mathrm{NO}_{2}^{-}$-примесей $N_{d}$ во всем исследованном диапазоне энергии накачки. Это указывает на то, что пакет неравновесных фононов распространяется в режиме диффузии (при квазидиффузии должна наблюдаться зависимость $\left.D^{-1} \sim N_{d}^{5 / 9}\left[{ }^{5}\right]\right)$. Таким образом, при интерпретации зависимости $D^{-1}\left(W_{\mathrm{H}}\right)$ следует исходить из модели нестационарной теплопроводности с сильным упругим рассеянием фононов на примесях.

Действительно, известно $\left[{ }^{6}\right]$, что стационарная теплопроводность кристаллов $\mathrm{KCl}-\mathrm{NO}_{2}^{-}$при гелиевых температурах определяется упругим рассеянием фононов на примесях, причем температурный ход коэффициента теплопроводности указывает на резонансный характер рассеяния с частотой $\omega$ рез $=20 \mathrm{~cm}^{-1}$. Детальный механизм резонансного рассеяния пока точно не установлен, однако влияние одноосного сжатия кристалла на скорость диффузии позволяет предполагать, что существенным является рассеяние фононов на переориентационных движениях примеси между эквивалентными положениями в кристалле-матрице [ $\left.{ }^{7}\right]$. Из сказанного следует, что коэффициент диффузии $D$ (по определению $D=\frac{1}{3} \bar{v}^{2} / \tau_{\text {pacc }}^{-1}$, где $\bar{v}-$ средняя скорость фононов, $\tau_{\text {расс }}^{-1}-$ суммарная вероятность различных процессов рассеяния) определяется главным образом вероятностью рассеяния фононов на $\mathrm{NO}_{2}^{-}$-примесях $\tau_{d}^{-1}(\omega)$, которое предполагаем состоящим из релеевской и резонансной частей

$$
\tau_{d}^{-1}(\omega)=N_{d}\left[A \omega^{4}+B \frac{\omega^{4}}{\left(\omega^{2}-\omega_{\mathrm{pe3}}^{2}\right)^{2}+\frac{\gamma^{2} \omega_{\mathrm{pe3}}^{2}}{4}}\right],
$$


где $N_{d}$ - концентрация примесей, $\gamma$ - ширина резонанса, $A$ и $B-$ константы релеевского и резонансного рассеяния соответственно. В предположении, что в нагретой области устанавливается локальная температура $T$, можем провести усреднение по распределению фононов $n(\omega)$, считая его планковским, и найти усредненную вероятность рассеяния $\overline{\tau_{d}^{-1}}$ как функцию от температуры

$$
\overline{\tau_{d}^{-1}}(T)=\int_{0}^{\omega_{D}} \tau_{d}^{-1}(\omega) n_{T}(\omega) \varrho(\omega) d \omega / \int_{0}^{\omega_{D}} n_{T}(\omega) \varrho(\omega) d \omega
$$

где $n_{T}(\omega)$ - число заполнения при температуре $T$, $\omega_{D}-$ дебаевская частота акустических фононов. С учетом (1), усредненный таким образом обратный коэффициент диффузии будет состоять из релеевской и резонансной частей $D^{-1}=D_{\text {рел }}^{-1}+D_{\text {рез }}^{-1}$ (рис. 1). Наилучшее согласие с экспериментом получается при выборе сотношения констант (см. (1)) $B / A=2,5 \cdot 10^{6} \mathrm{~cm}^{-4}$, что в области низких температур, где обе части являются релеевскими, дает соотношение вероятностей $B^{\prime} / A \sim 200$. Абсолютная величина константы $B \sim 10^{-9} \mathrm{~cm}^{3} / \mathrm{c}$, что согласуется с данными $\left[{ }^{4}\right]$.

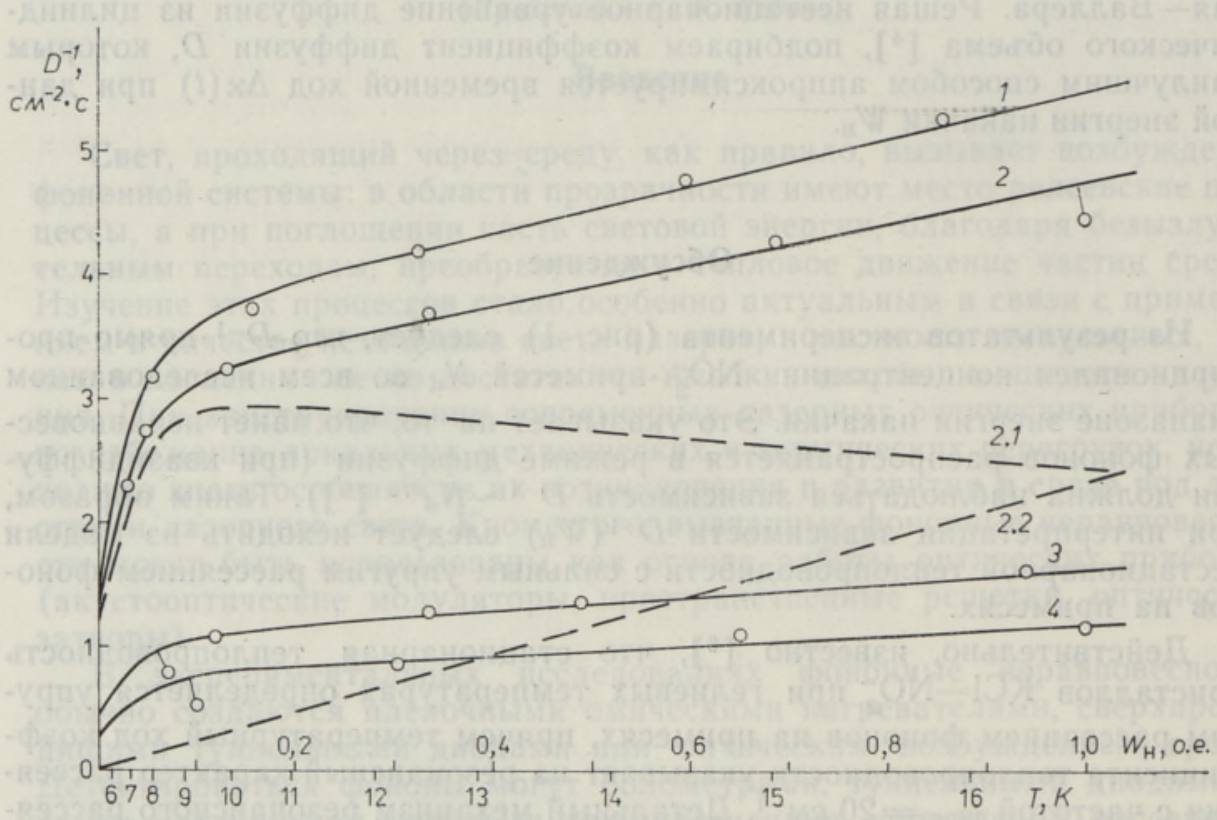

Рис. 1. Зависимость обратного коэффициента диффузии $D^{-1}$ от энергии накачки $W_{\text {н }}$ при различных концентрациях примесей:

$$
\begin{aligned}
& 1-N_{d} \equiv N_{0}=3 \cdot 10^{19} \mathrm{~cm}^{-3}, 2-N_{d}= \\
& =0,86 N_{0}, 3-N_{d}=0,30 N_{0}, 4-N_{d}=0,20 N_{0} \text {. }
\end{aligned}
$$

Кружками отмечены экспериментальные значения, сплошными линиями - модельные зависимости. Пунктиром показаны резонансная (2.1) и релеевская (2.2) составляıщие обратного коэффициента диффузии (кривая 2).

Обсудим наше предположение об установлении в возбужденном тракте локальной температуры. Критерием ее возникновения является условие $l_{0} \gg l(\bar{\omega})=\left[D(\bar{\omega}) \tau_{A}(\bar{\omega})\right]^{1 / 2}, \quad$ где $l_{0}-$ характерный размер возбужденной области, $\bar{\omega}-$ средняя частота фононного распределения, $\tau_{A}(\bar{\omega})$ 
Рис. 2. Зависимость $D^{-1}\left(W_{\text {н }}\right)$ для $N_{d}=0,86 \quad N_{0}$ в двойном логарифмическом масштабе. Пунктиром отмечена зависимость $W^{1 / 2}$.

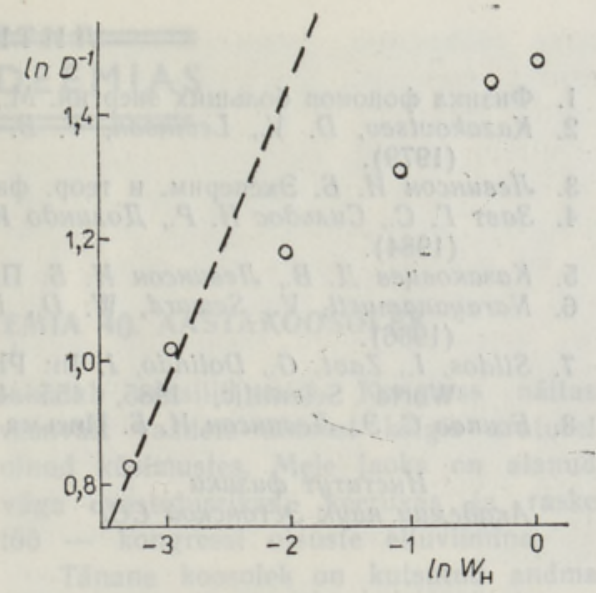

- время ангармонического распада «среднего» фонона, и следовательно, $l(\bar{\omega})$ - диффузионное смещение «среднего» фонона за время ангармонического взаимодействия. Данное условие означает, что «средний» фонон с частотой $\bar{\omega}$ не должен за время $\tau_{A}(\bar{\omega})$ выйти за пределы возбужденной области с линейными размерами $l_{0}$. В нашем случае $l_{0} \sim 0,1$ мм, и вычисления показывают, что $l(\bar{\omega})<0,1$ мм при $\bar{\omega}>8 \mathrm{~cm}^{-1}$. Это означает, что в тракте температура устанавливается для фононного пакета с $\bar{\omega}>8 \mathrm{~cm}^{-1}$, т. е. для значений $T>4 \mathrm{~K}$. В наших экспериментах нагрев тракта, определенный по фактору Дебая-Валлера, составлял 8-16 K (см. рис. 1), и таким образом, использование понятия температуры можно считать оправданным.

В связи с диффузионным характером распространения термализованного пакета фононов, а также с тем, что низкочастотные подтепловые фононы покидают тракт быстрее, чем расплывается основной пакет $\left[{ }^{4}\right]$, надо обсудить, не реализуется ли режим нелокальной фононной теплопроводности $\left[{ }^{3}\right]$. В этом режиме должна наблюдаться зависимость $D^{-1} \sim W_{\text {H. }}^{1 / 2}$. В наших экспериментах эта зависимость значительно слабее (см. рис. 2). Нами также установлено, что в тракте после возбуждения температура падает по закону $T \sim t^{-1 / 4}$. Это полностью согласуется с решением нестационарного уравнения диффузии фононов из цилиндрического объема. Заметим, что в режиме нелокальной фононной теплопроводности остывание тракта должно быть более быстрым $\left(T \sim t^{-10 / 21}\right)$ $\left[{ }^{8}\right]$.

Таким образом, зависимость скорости пространственного расплывания возбужденной лазерным импульсом горячей области от поглощенной энергии в кристалле $\mathrm{KCl}-\mathrm{NO}_{2}^{-}$объясняется диффузионной моделью, в которой предполагается сильное резонансное рассеяние на примесях $\mathrm{NO}_{2}^{-}$.

Авторы выражают глубокую благодарность Г. Завту за внимание к работе и ценные дискуссии. 


\section{Л ИТЕРАТ У РА}

1. Физика фононов больших энергий. М., «Мир», 1976.

2. Kazakovtsev, D. V., Levinson, Y. B. Phys. status solidi (b), 96, № 1, 117-127 (1979)

3. Левинсон Н. Б. Эксперим. и теор. физ., 79, вып. 10, 1394-1407 (1980).

4. Завт Г. С., Сильдос И. Р., Долиндо И. И. Физ. твердого тела, 14, № 5, 1424-1430 (1984).

5. Казаковщев Д. В., Левинсон И. Б. Письма в ЖЭТФ, 27, № 3, 194-196 (1978).

6. Narayanamurti, V., Seward, W. D., Pohl, R. O. Phys. Rev., 148, № 1, 481-494 (1966).

7. Sildos, I., Zavt, G., Dolindo, I. In: Phonon Physics (ed. J. Kollár et al.). Singapore, World Scientific, 1985, 455-457.

8. Есипов С. Э., Левинсон И. Б. Письма в ЖЭТФ, 34, № 4, 220 (1981).

\section{Ннститут физики}

Академии наук Эстонской ССР
Поступила в редакцию 2/VI 1986 